\title{
Editorial: Management of non-paralytic strabismus in infants, with special reference to esotropia
}

In the treatment of any defect of physiological function in the human body the aim should be to restore or develop, and maintain, a normal state of affairs as soon as possible or, failing this, to improve that function to the greatest possible extent. In strabismus there may be three defects of function which need correction:

(1) A defect of normal visual function of the squinting eye and in some cases of the non-squinting eye also; (2) a defect or loss of normal binocular function; (3) a physical deformity caused by the deviation of the visual axes, which in some cases may be quite minimal.

With regard to the first, no real problem is likely to exist if the squint is freely alternating or if it occurs only intermittently. But if the squint is unilateral and constant, especially if the deviation is longstanding and was manifest in earliest infancy, the loss of visual acuity in the squinting eye may be considerable and may be associated with noncentral fixation - an acquired and adaptive reaction and one which in general does not develop until a constant squint has been present for some time. So far as the second is concerned, this may develop as a result of a squint or in some cases it is possible that there is an underlying congenital defect of binocularity of heredofamilial origin. The third defect is the one that usually attracts the attention of parents, grandparents, or other observers, and its existence may be of the most profound importance because the physical deformity, if marked, may cause the parents and later the patient considerable social embarrassment. Indeed the squint itself and/or the defective visual acuity of a squinting eye may be a handicap in achieving certain forms of livelihood. It must, however, be appreciated that so far as visual acuity and binocular function are concerned a convergent squint of slight degree and not cosmetically obvious may be as pernicious in its effect on visual function as one of large degree.

In contradistinction it is interesting to note that normal or potentially normal binocular function is the rule rather than the exception in cases of primary divergent strabismus. This is probably because the deviation, at least in the early stages, is intermittent in nature or may occur only when viewing distant objects, being well controlled for near objects thanks to the power of convergence; or, less commonly, if there is an underlying convergence insufficiency, it may occur when viewing near objects only. Moreover, in exotropia any tangible degree of amblyopia is rare, since a diverging eye is invariably used for fixation when the patient is looking to the side of the deviation. Patients with a congenital divergent squint rarely get any worse if left untreated and invariably maintain their binocular potential. Hence the results of adequate surgical treatment, even if delayed until adult life, are usually most satisfactory. At any rate exotropia in children does not account for more than $15 \%$ of all cases of squint (R. M. Ingram, p. 4).

In cases of congenital ocular palsy of slight degree, including certain cases of the $\mathrm{A}$ and $\mathrm{V}$ syndrome and of Duane's retraction syndrome, it is amazing how normal binocular function is achieved and preserved by the adoption of a compensatory head posture and in the presence of a well-developed fusional reserve. These observations underline the innate desire for binocularity which appears to exist in most healthy infants. However, in squints of the convergent type with a fairly large deviation there is usually no position of the binocular gaze in which the infant can achieve binocular fixation. Hence the necessity for early and adequate treatment.

Although it is universally agreed that no child suffering from non-paralytic esotropia can be pronounced cured unless the visual acuity of each eye (with spectacle correction if needed) is of normal standard, and unless the ocular deviation has disappeared and the binocular function has become normal, yet in certain cases this ideal goal cannot be achieved, usually because the diagnosis of the condition has been made too late or the significance of the condition and the necessity for early treatment have not been fully realised.

Since esotropia of small angle may escape the notice of the average family doctor and yet may be associated with severe amblyopia in one eye, and since amblyopia due to anisometropia may exist in the presence of orthophoria, some form of screening of young children for visual defects caused by these conditions is most desirable.

As Ingram rightly points out, the treatment of amblyopia in a case of esotropia may have to await the recognition of the cosmetic deformity; and indeed only a little more than $50 \%$ of children with esotropia have a cosmetically noticeable deviation. He states that, although there is a first peak number 
of initial attendances of children with esotropia at the age of 3 or 4 years, there is also a second peak at the age of 5 years, when children first go to school and are subjected to a sight test. In his series he found that nearly $69 \%$ of cases of amblyopia presented after the age of 5 years and in three-quarters of these there was no detectable squint. Ingram therefore advocates an early screening test which is designed to identify both the squinter and the straight-eyed amblyope, for there is no doubt that the earlier a firm diagnosis of squint and/or amblyopia is made and the earlier the corrective treatment is applied the better the prognosis. The present system of vision testing on school entry (at 5 years of age) is obviously inadequate, and even if subjective testing of vision is made, as it can be in co-operative children of $2 \frac{1}{2}$ or 3 years of age, it may not give sufficiently accurate results to be of value. It also has the disadvantage of being very time-consuming.

Ingram therefore suggests that an examination of the refraction carried out under a cycloplegic should be used as a quick objective method of screening. The reason for this is based on the clinical research he has done, which shows: (1) +2.0 to $+2.75 \mathrm{D}$ of spherical hypermetropia in the more emmetropic of a pair of hypermetropic eyes is significantly associated with esotropia, and this is so even in the absence of anisometropia. It is also significantly associated with some degree of amblyopia irrespective of whether esotropia is present. This information is probably the first definite attempt to define what is an abnormal amount of hypermetropia in a child. (2) Anisometropia is significantly associated with esotropia, but only if cases of spherical hypermetropia of $+2.0 \mathrm{D} \mathrm{Sph}$ or more in the more emmetropic eye are included. (3) In all cases of esotropia the added presence of anisometropia is significantly associated with an increased chance that the child will also become amblyopic. Ingram states that no improvement in the treatment of straight-eyed amblyopes nor of esotropia not cosmetically noticeable can be expected unless visual acuity can be adequately tested well before school entry, or some special screening test, e.g., refraction, is carried out. In routine screening procedures by objective refraction in 209 children of the preschool age (mainly between 1 and 2 years) who were found to have esotropia, $72 \%$ had an abnormal refraction. We await with interest the results of Ingram's treatment of his series of cases in terms of visual acuity and of binocular function.

There is no doubt that children who are 'at risk' with regard to squint and amblyopia should be subjected to early-eye examination including refraction. This refers to those families in which there is a history of 'squint', 'cast', 'turn', or 'lazy eye' in a child's mother, father, grandparents, siblings, aunts, uncles, or cousins or a history of any of these relations having had to wear glasses since early childhood. The paper by E. Chimonidou (p. 26), of Athens, supports the hereditary nature of strabismus which dates from early infancy. In the series of cases of squint seen in Greece $81.2 \%$ were convergent and $18.8 \%$ divergent, and in those suffering from amblyopia a satisfactory result (equal and normal vision in each eye) resulted in $60 \%$ of cases. These authors considered that a refractive error was significant when hypermetropia exceeded $3.0 \mathrm{D}$, when myopia of any degree was present, or when astigmatism was over $0.50 \mathrm{D}$ or anisometropia over $1.0 \mathrm{D}$. No data are given as to binocular function.

The findings in the paper by Higham (p. 30) on stereopsis and aniseikonia in uniocular aphakia, although dealing with adults, should also apply to children. He finds that when aphakia is corrected with a contact lens it is in most patients no obstacle to binocular function. The comfort of the contact lens is, of course, an important factor. In the 27 uniocular aphakics he examined there were only 2 with no demonstrable stereopsis on the Titmus chart. The aniseikonia in these 2 patients was 25 and $29 \%$, respectively; in all the other patients the aniseikonia was below $20 \%$. In children with uniocular aphakia it would appear to be advisable to overcorrect the contact lens by $+3 \mathrm{D}$ and give the patient spectacles to neutralise this for distance.

The treatment of amblyopia first comprises full correction of the refraction. If this controls the deviation, and if it can be shown that normal retinal correspondence and fusion with a reasonable range is present, the constant wearing of glasses will in due course gradually lead to improvement of visual acuity of the amblyopic eye. The process can be hastened by part-time occlusion of the betterseeing eye. If, however, no binocular function can be demonstrated, constant occlusion of the betterseeing eye is indicated; and, once occlusion has achieved equal vision in each eye both for distance and near, regular occlusion of one or other eye should be practised until steps are taken to give the patient sufficient parallelism of the visual axes to enable him to obtain stimulation of the two foveae by images of the same object. This may be achieved by accurate surgical treatment, which may be needed in more than one stage or in the first instance by the use of prisms, aided, if indicated, by the use of orthoptic exercises.

Dr Pigassou in her paper (p. 16) with its inviting title of 'Entente cordiale in the early treatment of squint' questions the validity of Kenneth Wybar's statement (presumably with regard to the surgical 
treatment of esotropia in patients between the age of 5 and 6 years) that he regards as a highly satisfactory result a slightly anomalous type (of retinal correspondence), so that the condition represents a secondary form of microtropia. She believes that 'to cure a squinter is to give him normal binocular vision'. This may be so, but, if a small angle of deviation with anomalous fusion, a good range of fusion, and full stereopsis is achieved, the result is highly satisfactory to the patient and his parents and appears to give permanent stability. Prismotherapy as practised by Dr Pigassou is essentially preoperative and is accompanied by exercises to establish single binocular vision in artificial conditions followed by the development of fusion and then stereoscopic vision. If, initially, retinal correspondence is normal, exact prisms to correct the deviation are used. If, however, retinal correspondence is abnormal, overcorrecting prisms with an overcorrection of about 15 dioptres are used with change of the fixating eye.

In discussing the aim of early treatment $\mathrm{Dr}$ Pigassou points out that binocular vision is a conditioned reflex which develops in the first years of life, and if some factor causes an ocular deviation this will produce abnormal adaptations which must be destroyed before they become deep-rooted. She emphasises the importance of specially adapted orthoptic examination and treatment to suit the intellectual and sensory-motor capacities of the child, and the need for the full collaboration of the parents. She reports that the results of prismotherapy are amazingly good $-100 \%$ of cures in children correctly treated. (Presumably those whose parents lacked the necessary co-operation to see that the treatment was correctly carried out were not included in the results.) She concludes that: (1) Thanks to early treatment, we should encounter in the future neither eccentric fixation nor anomalous retinal correspondence. (2) The functional treatment of strabismus should be begun between 1 and 3 years of age (this opinion is in line with current therapy of all developmental troubles). (3) Early treatment is possible only if the early detection of squint is organised and if appropriate techniques are used.

Similar statements were made by Dr E. Thomas 52 years ago in his opening paper at the Oxford Ophthalmological Congress of 1924. He said with regard to the treatment of convergent concomitant strabismus, 'Once we have the parents made to understand the importance of instant ... attention to squint at whatever age it occurs we shall be in a fair way, I hope and think, to reduce the incidence of squint and amblyopia and shall have moved a step forward towards the ideal of prevention.... And when I have suggested a still higher ideal of prevention by the, preferably expert, examination of children before entering school I have done what I set out to do.' 